\title{
The Interpretation of the Radarograms on the Basis of Experimental Data
}

\author{
M. Zhartybayeva*, Z. Oralbekova And K. Iskakov
}

L.N. Gumilyov Eurasian National University, Department of Information Technologies, Astana, Kazakhstan

\begin{abstract}
An algorithm for solving the inverse problem of subsurface radiolocation using data from ground penetrating radar is presented in this paper. Experimental research was carried out using the geophysical device "Loza B" in field conditions; a part of airfield runway, located on the territory of Almaty region (Kazakhstan), was investigated. The conclusion concerning the reasons of occurrence of defects on the surface of the runway is given.
\end{abstract}

DOI: 10.12693/APhysPolA.128.B-467

PACS: 73.43.fj, 73.43.Fj, 01.50.hv

\section{Introduction}

Ground penetrating radar, commonly called GPR or Georadar, is a geophysical devise measuring the travel time of radio-waves. At the boundary of the medium an electromagnetic pulse is generated by a transmitter and antenna (GPR), and at the same border the response of the medium, as a set of waves with different travel time, intensity and shape is captured by the receiving antenna and is recorded by the receiver.

The inverse problem of subsurface radiolocation is the restoration of the structure of the underground medium using GPR data (the response of the medium).

In this work the surface of the airfield runway, located on the territory of Almaty region in Kazakhstan, was probed using a geophysical complex "Loza B" with antenna of $150 \mathrm{~cm}(150 \mathrm{MHz})$ and a profile step of $20 \mathrm{~cm}$. The differences of dielectric permittivity and conductivity between different geological rocks incline us to use a georadar in this study.

\section{Experimental research}

\subsection{Description of the objective and its physical statement}

The objective of this work was (i) to identify the internal structure of the site on the airfield runway, with an obvious defect, presented in Fig. 1, (ii) survey of ground structure in order to detect the reasons of the defect of the airstrip surface. The statement of the problem requires the use of non-destructive method, which gives an idea about the state of the soil located beneath the working surface of the airstrip.

Experimental research was conducted by safety airfield service director B. Kulnazarov, professor K.T. Iskakov, Zh.O. Oralbekova and M.G. Zhartybayeva (Fig. 1) [1].

The experimental study consisted of the following parts: a description of the studied object and its physical

*corresponding author; e-mail: makkenskii@mail.ru

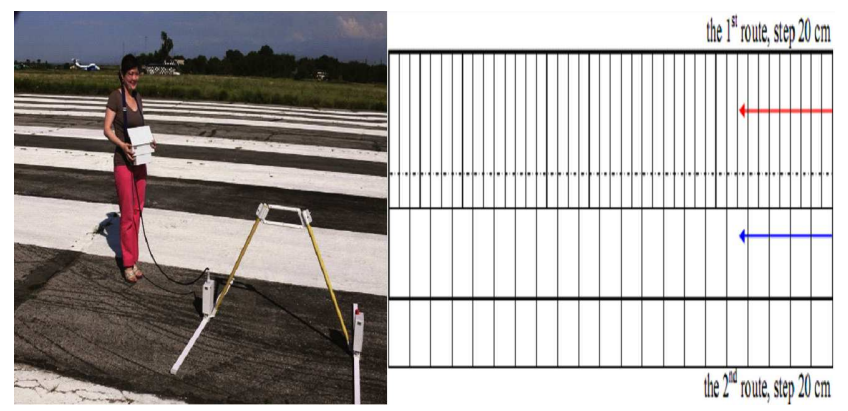

Fig. 1. Runway, georadar and the scheme of the runway.

condition, design of the partitioning scheme of the portion of the band for the GPR measurements, acquiring geological profiles data of the studied object, presenting an expert conclusion concerning the defect of the studied object.

Experimental investigation was performed using the geophysical complex "Loza B" along two routes, using the antenna of $100 \mathrm{~cm}(100 \mathrm{MHz})$, with $10 \mathrm{~cm}$ step along the profile on the first route (in Fig. 1 marked with a red arrow), and also using the antenna of $150 \mathrm{~cm}(150 \mathrm{MHz})$ with step of $20 \mathrm{~cm}$ on the second route (marked in Fig. 1 with blue arrow).

\subsection{Georadar data of experimental research facility}

Typically, the GPRs have the embedded software, the output data of which is the so-called radarogram [1]. Figure 2 shows the profile along the route shown in Fig. 1 with a red arrow, produced using "Krot179Ns" program. The values from left to right along the horizontal axis in Figs. 2, 3 represent the coordinates in meters, measured along the profile, while the vertical scale on the right represents the time delay between sending and receiving the signal. Vertical scale on the left shows the distance in meters traveled by the signal, assuming that the speed of the signal is the same as that of the surface layer. This distance is not related to the depth of the layers. 


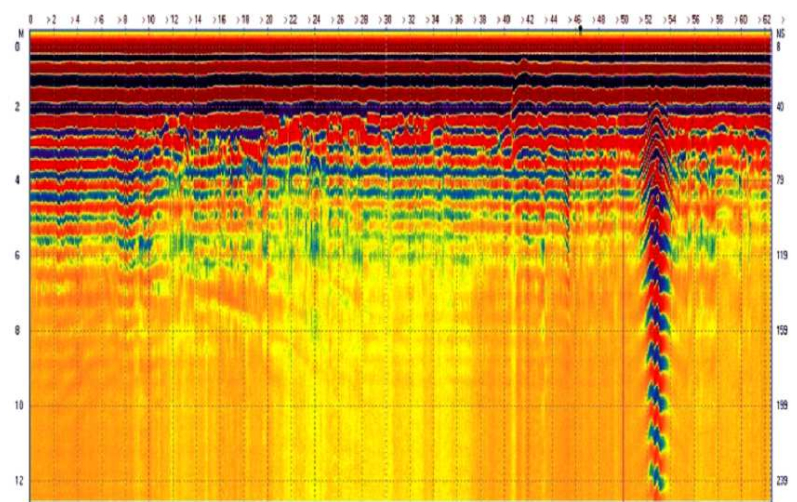

Fig. 2. Profile slopes in increments of $10 \mathrm{~cm}$, for a distance of $100 \mathrm{~cm}$ between the antennas.

Here are some similar images (Georadar profile) recorded along the second route (see Fig 3).

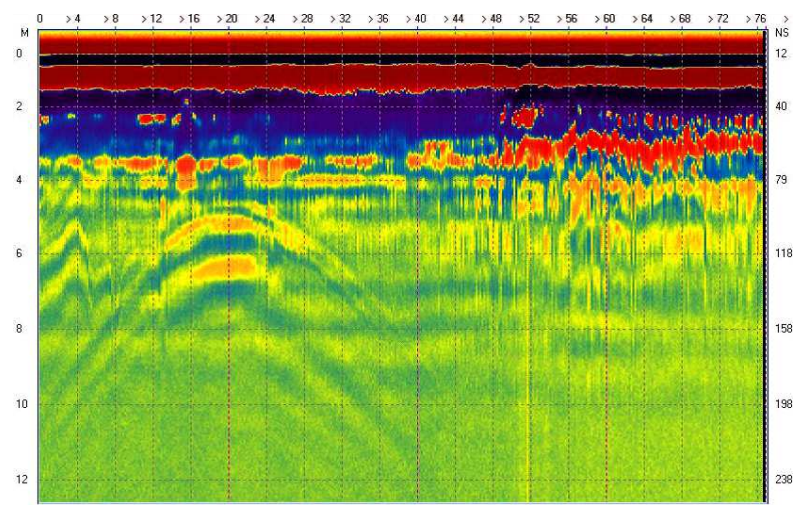

Fig. 3. Profile of the route with step of $20 \mathrm{~cm}$, for a distance of $150 \mathrm{~cm}$ between the antennas.

In both radarograms can be observed a pattern characteristic of the reflection from the underground facilities such as pipes (dome-shaped wave pattern). Particularly clear picture of the reflection is visible on the second route, where the top of the dome is $20 \mathrm{~m}$ from the startline. Along the first route the summit of the dome in the radarogram is located at $12 \mathrm{~m}$. Therefore, a pipe passes diagonally under the runway.

\section{Conclusions}

Pictures of radarograms are visually similar to the perturbation of the liquid surface by a plane wave front of which coincides with the "shoreline". Grounds for ongoing hydrodynamic analogy here are as follows:

1. The propagation model of electromagnetic oscillations in a medium that is used as the base model in georadar, coincides with equations of wave propagation of the liquid surface. In both cases we are dealing with wave phenomena.
2. Radarogram are obtained when recording medium response to a disturbance, when moving the source along the route. The distance between the receiving and emitting antennas is significantly smaller than the length of the route.

3. Imagine that we have disturbed the medium at the same time between the sources placed at all points of the route (which in fact we passed in succession, one after the other). That is, as if we simultaneously included as many units as the measuring points were on the road.

4. This simultaneous perturbation of multiple sources can be treated as a perturbation of the surface of the medium by a plane wave incident on the surface.

If on the way of the waves there are impermeable barriers, the waves bend around them and there is a domeshaped wave pattern (Fig. 2 and 3), which is similar to the wave pattern that is formed by a stone protruding from the water, for example.

If the obstacles are something like a gate, then the wave passes through the gate, and outside it is calm. In Fig. 2 at a distance interval of $52-54 \mathrm{~m}$ from the origin, there is a kind of such "gateway" or rather "doors".

These considerations allow us to draw conclusions about the presence of a "pipe", an obstacle impervious to electromagnetic oscillations. Reason for this is that radarogram pattern is similar to the wave pattern in the vicinity of the rock in the water, using our hydrodynamic analogy, proposed here.

On the same grounds, we conclude that there is a possibility of a "gap", a narrow region below the first-run route, which is transparent to radio waves.

Conclusions about the extinction of the signal are made only on the basis of attenuation of the reflected signal and the comparison with adjacent sections of the radarogram. In spots where the extinction is stronger, there is more conductivity and, therefore, likely there is humid environment [2].

\section{References}

[1] S.I. Kabanikhin, K.T. Iskakov, Zh.O. Oralbekova, Proceedings of the $8^{\text {th }}$ Congress of International Society for Analysis, its Applications, and Computation (ISAAC-2011), Moscow, Russia, p. 291.

[2] K.T. Iskakov, B.G. Mukanova, Zh.O. Oralbekova, Proceedings of the third International Scientific School Conference "Theory and numerical methods for solving inverse and ill-posed problems", Novosibirsk. 